\title{
Conhecimentos e atitudes de cirurgiões-dentistas da rede pública de Pelotas-RS frente aos maus- tratos infantis
}

\author{
Giulia Tarquinio Demarco*, Ivam Freire da Silva-Júnior ${ }^{* *}$, Marina Sousa Azevedo*** \\ * Mestranda, Programa de Pós-Graduação em Odontologia, \\ Faculdade de Odontologia, Universidade Federal do Rio \\ Grande do Sul \\ ** Mestre, Programa de Pós-graduação em Odontologia, \\ Faculdade de Odontologia, Universidade Federal de Pelotas \\ *** Professora Adjunta, Faculdade de Odontologia, \\ Universidade Federal de Pelotas
}

Recebido: 10/12/2020. Aprovado: 18/05/2021.

\begin{abstract}
RESUMO
O presente estudo objetivou identificar e analisar os conhecimentos e as atitudes dos cirurgiõesdentistas (CD) da rede pública de Pelotas-RS frente aos maus-tratos infantis (MTI). Os dados foram coletados por um questionário semiestruturado. Coletaram-se informações sociodemográficas, relativas ao trabalho, conhecimentos e atitudes dos $\mathrm{CD}$. Realizou-se uma análise estatística descritiva e associações entre as variáveis independentes com os conhecimentos e atitudes por meio do teste de Exato de Fisher e Qui-quadrado $(\mathrm{p}<0,05)$. Dos 45 CD incluídos na amostra, mais da metade afirmaram nunca ter recebido informações sobre o tema, 71,1\% entenderam ser responsabilidade do CD a identificação de casos suspeitos, $50 \%$ não souberam informar a respeito da implicação legal sobre a falta de notificação e $86,7 \%$ acreditaram que se deve avisar ao médico ou enfermeiro para que eles tomem alguma atitude. Observou-se que $60 \%$ dos profissionais nunca suspeitaram de um caso e $25 \%$ dos que suspeitaram tomaram alguma atitude. Houve associação estatística entre a responsabilidade do $\mathrm{CD}$ e o sexo e o tempo de formação, da atitude correta frente a um caso suspeito e a pós-graduação, e ainda, da implicação legal com a idade e tempo de formação. Os conhecimentos e atitudes dos CD sobre MTI foram melhores entre profissionais mais jovens, do sexo feminino, com menor tempo de formados, possuindo ou cursando alguma pós-graduação. Os CD conhecem sua importância frente a essa violência, porém apresentam dificuldades quanto às atitudes a tomar. Descritores: Maus-tratos Infantis. Violência. Odontologia Legal. Educação em Odontologia.
\end{abstract}




\section{INTRODUÇÃO}

A violência é uma temática enrustida na sociedade há muito tempo, porém atualmente vem ganhando visibilidade. Na década de 1990, houve a criação do Estatuto da Criança e do Adolescente (ECA), sob a lei $\mathrm{n}^{\circ} .8 .069$, onde está descrito que "nenhuma criança ou adolescente será objeto de qualquer forma de negligência, discriminação, exploração, violência, crueldade e opressão, punido na forma da lei qualquer atentado, por ação ou omissão, aos seus direitos fundamentais". Todavia, milhares de crianças têm esse direito violado constantemente, visto que os maus-tratos infantis (MTI) são considerados um problema de saúde pública no Brasil e no mundo ${ }^{2-4}$.

Os MTI podem ser referidos como violência doméstica, violência infantil ou abuso infantil e se caracterizam como qualquer ato ou omissão dos pais ou responsáveis, indivíduos em condição de superioridade, capazes de provocar injurias de físicas, psicológicas ou sexuais à criança ou ao adolescente ${ }^{5}$. Esse tipo de violência deixa as vítimas mais propensas ao desenvolvimento de outros problemas ao longo de sua infância e vida adulta, variando desde transtornos físicos até consequências psicológicas, como depressão e a baixa autoestima ${ }^{6}$.

Os CD estão em uma posição considerada de privilégio para identificação e descoberta dos sinais dessa violência ${ }^{7,8}$, visto que grande parte das lesões resultantes da agressão ocorrem na região de cabeça e pescoço ${ }^{3,7,9}$. Portanto, é essencial que estes profissionais recebam o treinamento apropriado, que os permita identificar os MTI e prosseguir com as condutas corretas ${ }^{10}$.

É importante ressaltar que os profissionais da saúde têm a obrigatoriedade de notificar aos órgãos competentes casos suspeitos ou confirmados, sob risco de penalização ${ }^{11}$. Constata-se que a partir da atitude de notificar pode-se criar um vínculo entre duas partes distintas do sistema, que são o serviço de atenção a saúde e o sistema judicial ${ }^{12}$. Dentro desse contexto, cabe ressaltar, sobre a intersetorialidade nos processos de trabalho em saúde, que pode ser considerada como elemento importante para a promoção de saúde. Sendo assim é fundamental, para o funcionamento dessa dinâmica ocorra, que os seus vários setores e componentes mantenham esse contato próximo, dialogando entre si, com o intuito de suprir necessidades da comunidade e promover seu bem estar $^{13}$.

Todavia, apesar de diversos direitos, leis, equipes multiprofissionais, obrigatoriedade de notificação e posição privilegiada dos dentistas, ainda está presente uma subnotificação dos $\operatorname{casos}^{14}$. Há estudos que associam o despreparo dos profissionais em lidar com essas vítimas que vão aos serviços de saúde à ausência deste conteúdo na graduação ${ }^{9,15}$. Associa-se essa dinâmica a outros fatores, como o medo de envolvimento e por ser uma temática ainda pouco explorada, o que pode impactar negativamente na precisão para a identificação da violência e das atitudes frente a casos suspeitos de MTI $^{16}$.

As Unidades Básicas de Saúde (UBS), por serem a porta de entrada principal para os usuários do Sistema Único de Saúde (SUS) e por integrarem profissionais de variadas áreas que trabalham em equipe, são um ambiente propício para a atuação efetiva e eficaz frente a um caso de MTI $^{17}$. Assim, o objetivo deste estudo foi descrever os conhecimentos e as atitudes dos CD atuantes nas UBS de Pelotas-RS frente aos casos de MTI e verificar a relação destes conhecimentos e atitudes com os aspectos sociodemográficos e do processo de trabalho desses profissionais.

\section{MÉTODOS}

Trata-se de um estudo transversal, aprovado pelo Comitê de Ética em Pesquisa da Faculdade de Odontologia da Universidade Federal de Pelotas (Parecer 3.282.976, CAAE 11600219.7.0000.5318).

O cenário para condução do estudo foi a 
cidade de Pelotas-RS, onde a população-alvo foi constituída pelos CD atuantes em UBS, caracterizando uma amostra de conveniência. Portanto, o critério de inclusão no estudo foi ser um CD vinculado à Rede de Atenção Básica do município de Pelotas. Foram excluídos aqueles profissionais que possuíam cargos de gestão, que eram atuantes somente em programas de promoção de saúde nas escolas ou em Centros de Especialidades Odontológicas (CEO).

Os dados foram coletados por meio de um questionário semiestruturado, autoaplicado e anônimo. Todos os profissionais que aceitaram participar da pesquisa assinaram o Termo de Consentimento Livre e Esclarecido. O questionário possuía 26 questões, 3 abertas e 23 fechadas, divididas em três domínios analíticos: os dados sociodemográficos e de formação profissional (bloco A), os conhecimentos adquiridos acerca da temática e importância (bloco B) e as atitudes frente a casos suspeitos de MTI (bloco C).

No bloco A coletou-se informações quanto ao sexo, idade, tempo de formação, modelo de atenção, rotina de trabalho e pós-graduação (mestrado e doutorado). As variáveis idade e o tempo de formação foram coletadas em anos e dicotomizadas por meio da sua mediana. O modelo de atenção da UBS foi categorizado em Estratégia Saúde da Família (ESF), mista (possuía 2 processos de trabalho ESF e Tradicional) e tradicional (sem a presença da equipe da ESF). O pertencimento do $\mathrm{CD}$ também foi categorizado em fazer parte da ESF e não fazer parte ou estar em UBS tradicional, a qual não tinha essa equipe. A rotina de trabalho do profissional foi categorizada em atuação apenas na esfera pública ou dividindo sua rotina de trabalho entre o serviço público e privado. A pós-graduação (mestrado e doutorado) foi dicotomizada em estar cursando ou ter realizado e não estar cursando ou não possuir o título.

No bloco B foram coletadas as variáves sobre conhecimento do $\mathrm{CD}$ frente ao tema, se haviam e onde haviam adquirido informações sobre o tema, a capacidade e responsabilidade na identificação dos casos e a ação frente a uma suspeita, assim como da obrigatoriedade em notificar (implicação legal). A capacidade de identificação dos casos de MTI foi mensurada por meio de uma escala de 0 a 10, na qual o valor de zero era nada capaz e dez era totalmente capaz. Em relação à ação diante de uma suspeita, para ser considerada correta, deveria-se responder que notificaria o caso aos órgãos competentes, podendo ainda associar-se com o registro no prontuário e manter a criança em acompanhamento. Ainda neste bloco, questionou-se sobre o recebimento de capacitação sobre o tema, e caso não tivesse, se desejaria receber; o conhecimento sobre as fichas de notificação; a confiança nos órgãos de proteção e sobre a discussão de MTI na UBS e entre os colegas de trabalho.

No bloco $\mathrm{C}$, das atitudes frente a casos de MTI, englobaram-se variáveis relativas a ter havido suspeita do profissional, se havia sido o primeiro a suspeitar do caso e como havia sido a conduta. Sobre a conduta frente a um caso foi categorizado em correto quando o CD notificou o caso aos órgãos competentes e buscou acompanhamento do indivíduo nos casos já notificados; para as demais respostas classificou-se como incorreto, ou sem suspeita alguma para aqueles que nunca haviam suspeitado.

A aplicação do questionário ocorreu nos momentos das capacitações e/ou reuniões de trabalho oferecidas pela Prefeitura Municipal de Pelotas, que reúne os $\mathrm{CD}$ da rede pública do município. Uma estudante de graduação previamente treinada foi responsável pela supervisão da aplicação, explicação sobre a pesquisa e esclarecimento de quaisquer dúvidas. A coleta de dados foi realizada de maio a outubro de 2019. O profissional que não compareceu a pelo menos três tentativas de encontro nas reuniões/capacitações foi considerado como perda. 
Os dados foram codificados e tabulados duplamente em planilha e exportados para o software Stata 12.0 (StataCorp, College Station, TX, EUA). Foi realizada a análise estatística descritiva, na qual foram observadas as variáveis conforme suas frequências relativas e absolutas. Utilizaram-se os testes Exato de Fisher e Quiquadrado para identificar associações das questões relativas aos CD e da UBS (dados sociodemográfico e de trabalho) com os conhecimentos sobre a sua responsabilidade na identificação de MTI, como agir e a implicância legal da não tomada de atitude, assim como com as atitudes frente aos casos de MTI. Os dados foram considerados como estatisticamente significantes quando obtiveram um valor de $\mathrm{p}<0,05$.

\section{RESULTADOS}

Dos $55 \mathrm{CD}$ vinculados à rede pública, dois profissionais foram excluídos, por serem atuantes em cargos de gestão. Dos 53 CD elegíveis, atuantes em 37 UBS das 42 existentes em Pelotas, oito constituíram-se como perdas devido às suas ausências em reuniões ou licenças médicas ou de maternidade. Portanto, 45 profissionais da rede pública de Pelotas participaram do estudo. Não houve recusa em participar.

$\mathrm{Na}$ tabela 1 observa-se a caracterização dos CD com relação aos aspectos demográficos, de formação profissional e sobre o serviço no qual estão inseridos. Pode-se observar que a amostra foi preponderantemente do sexo feminino $(88,9 \%)$, na faixa etária entre 28 e 42 anos (57,8\%), com até 16 anos de formado $(53,3 \%)$, com rotina de trabalho dividida entre os setores público e privado $(71,1 \%)$ e que não possuíam pós-graduação stricto sensu (75,6\%). Com relação à caracterização do serviço, a grande maioria das UBS tinham modelo de atenção misto $(42,2 \%)$ e $62,2 \%$ dos profissionais não pertenciam a ESF ou trabalhavam em um modelo tradicional, que não possui a equipe em sua contituição.

Tabela 1. Caracterização dos cirurgiões-dentistas e do serviço da rede municipal de saúde de Pelotas (n=45)

\begin{tabular}{lcc}
\hline Variável & n & $\%$ \\
\hline Sexo & 40 & 88,9 \\
$\quad$ Feminino & 5 & 11,1 \\
$\quad$ Masculino & & \\
Idade (anos) & 26 & 57,8 \\
$28-42$ & 19 & 42,2 \\
$\quad 43-69$ & & \\
Tempo de formado & 24 & 53,3 \\
$\quad$ Até 16 anos & 21 & 46,7 \\
$\quad$ Maior que 16 anos & 15 & 33,3 \\
Modelo de Atenção da UBS & 19 & 42,2 \\
$\quad$ ESF & 11 & 24,5 \\
$\quad$ Misto & & \\
$\quad$ Tradicional & 28 & 62,2 \\
Dentistas pertencentes a ESF & 17 & 37,8 \\
$\quad$ Não/Tradicional & & \\
$\quad$ Sim & 13 & 28,9 \\
Rotina de Trabalho & 32 & 71,1 \\
Integralmente público & & 75,6 \\
$\quad$ Público/Privado & 34 & 24,4 \\
Pós-graduação Stricto Sensu & 11 & \\
$\quad$ Não possui & & \\
Possui ou está cursando &
\end{tabular}


$\mathrm{Na}$ tabela 2 demonstra-se os No que se refere à implicação legal para o $\mathrm{CD}$ em conhecimentos sobre MTI dos CD da rede caso de não notificação, 43,2\% acreditaram pública da cidade de Pelotas. Mais de metade dos haver e 50\% não souberam informar. Quase a CD afirmou nunca ter recebido informações totalidade da amostra (97,8\%), nunca haviam sobre o tema na graduação $(55,6 \%)$ e na atuação participado de qualquer tipo de capacitação ou profissional $(53,3 \%)$. Sobre a responsabilidade treinamento após seu ingresso no serviço do CD em identificar casos de MTI, 71,1\% deles público. Nenhum participante referiu conhecer a entenderam ser responsabilidade do profissional. ficha de notificação.

Tabela 2. Conhecimentos sobre maus-tratos infantis dos cirurgiões-dentistas da rede pública da cidade de Pelotas $(n=45)$

\begin{tabular}{|c|c|c|}
\hline Variável & $\mathbf{n}$ & $\%$ \\
\hline \multicolumn{3}{|c|}{ Informações durante a Graduação } \\
\hline Não & 25 & 55,6 \\
\hline Sim & 16 & 35,5 \\
\hline Não sei/Não lembro & 4 & 8,9 \\
\hline \multicolumn{3}{|c|}{ Informações durante a Atuação Profissional } \\
\hline Não & 24 & 53,3 \\
\hline Sim & 19 & 42,2 \\
\hline Não Sei/Não lembro & 2 & 4,5 \\
\hline \multicolumn{3}{|c|}{ Responsabilidade do cirurgião-dentista } \\
\hline Não & 5 & 11,1 \\
\hline Sim & 32 & 71,1 \\
\hline Não sei & 8 & 17,8 \\
\hline \multicolumn{3}{|c|}{ Implicância legal da não notificação* } \\
\hline Não & 3 & 6,8 \\
\hline Sim & 19 & 43,2 \\
\hline Não sei & 22 & 50,0 \\
\hline \multicolumn{3}{|c|}{ Capacitação sobre MTI } \\
\hline Não & 44 & 97,8 \\
\hline Sim & 1 & 2,2 \\
\hline Não sei/Não lembro & - & - \\
\hline \multicolumn{3}{|c|}{ Conhecimento da ficha de notificação } \\
\hline Não & 45 & 100 \\
\hline Sim & - & - \\
\hline Não sei & - & - \\
\hline \multicolumn{3}{|c|}{ Confiança nos órgãos* } \\
\hline Não & 3 & 7,0 \\
\hline Sim & 22 & 51,2 \\
\hline Não sei & 18 & 41,8 \\
\hline \multicolumn{3}{|c|}{ Discussão de MTI na UBS } \\
\hline Não & 17 & 37,8 \\
\hline Sim & 18 & 40,0 \\
\hline Não sei/Não lembro & 10 & 22,2 \\
\hline
\end{tabular}


Relativo à questão de quanto os CD se consideram capazes de identificar casos de MTI, constatou-se que a média de 5,65 $\pm 2,1$ (de 0 a 9), com a maior parte dos participantes $(27,9 \%)$ definindo sua capacidade de identificação em um valor de 5 .

No gráfico 1, sobre como agir diante de um caso de suspeita de MTI, quando os profissionais poderiam registrar mais de uma opção de resposta, constatou-se que a grande maioria deles $(86,7 \%)$ acreditou que se deva avisar ao médico ou enfermeiro sobre um caso suspeito para que estes tomem atitude.

Considerando as condutas corretas a serem tomadas, $48,9 \%$ acreditavam que se deva notificar aos órgãos competentes, $60 \%$ consideraram correto buscar manter essa criança em acompanhamento no serviço e $60 \%$ presumiram ser correto o registro no prontuário.

Referente ao domínio analítico das atitudes observou-se que $60 \%(\mathrm{n}=27)$ dos profissionais nunca suspeitaram de nenhum caso de MTI. Dentre os que suspeitaram ( $\mathrm{n}=18), 15$ não foram o primeiro profissional a levantar essa suspeita e 11 tomaram algum tipo de atitude frente ao caso suspeito (tabela 3).

Com relação às condutas tomadas, estas foram relatadas por meio de uma questão aberta. Constatou-se que a grande maioria dos CD encaminhou ou conversou com os outros profissionais (médico, enfermeiro e assistente social) para que estes tomassem alguma conduta. Houve um caso em que a suspeita foi referida à instituição escolar na qual a criança estudava. Por fim, houve dois profissionais que relataram que os seus casos já haviam sido notificados aos órgãos competentes e estavam em acompanhamento com médico e enfermeiro e outros dois casos em que foi realizada a notificação junto ao órgão competente.

$\mathrm{Na}$ tabela 4 estão apresentadas as associações entre os dados sociodemográficos e de trabalho com as questões de conhecimento dos CD. Houve associação estatística entre resposta correta ao questionamento sobre a responsabilidade do $\mathrm{CD}$ e $\mathrm{o}$ sexo dos profissionais, tendo $77,5 \%$ das profissionais do sexo feminino e $20 \%$ do sexo masculino respondendo corretamente. Observou-se ainda significância estatística entre a resposta correta referente à responsabilidade do dentista e o tempo de formado: $87,5 \%$ dos CD que haviam se formado há menos de 16 anos responderam corretamente sobre a conduta a se tomar, contra $52,4 \%$ daqueles que se formaram há mais tempo.

Houve associação estatística entre o conhecimento a respeito da ação correta frente a um caso suspeito de MTI (considerando-se aqueles que responderam que fariam a notificação ao órgão de proteção) e possuir pósgraduação (mestrado e doutorado), $81,8 \%$ dos profissionais que já possuem ou estão cursando esse nível de formação responderam corretamente, em comparação a 38,2\% dos que não possuíam esse tipo de titulação.

Referente à alternativa correta quanto ao conhecimento sobre a implicação legal ao CD que não notifica um caso suspeito de MTI, foi verificada associação estatística com a idade dos profissionais e com o tempo de formado. Os dentistas que estão na faixa etária de 28 a 42 anos de idade (mais jovens) tiveram uma taxa de acerto maior (56\%), assim como aqueles com menor tempo de formados $(61,9 \%)$.

Não foi observada associação entre os dados sociodemográficos e de trabalho e as atitudes dos profissionais frente aos casos suspeitos de MTI (tabela 5). 


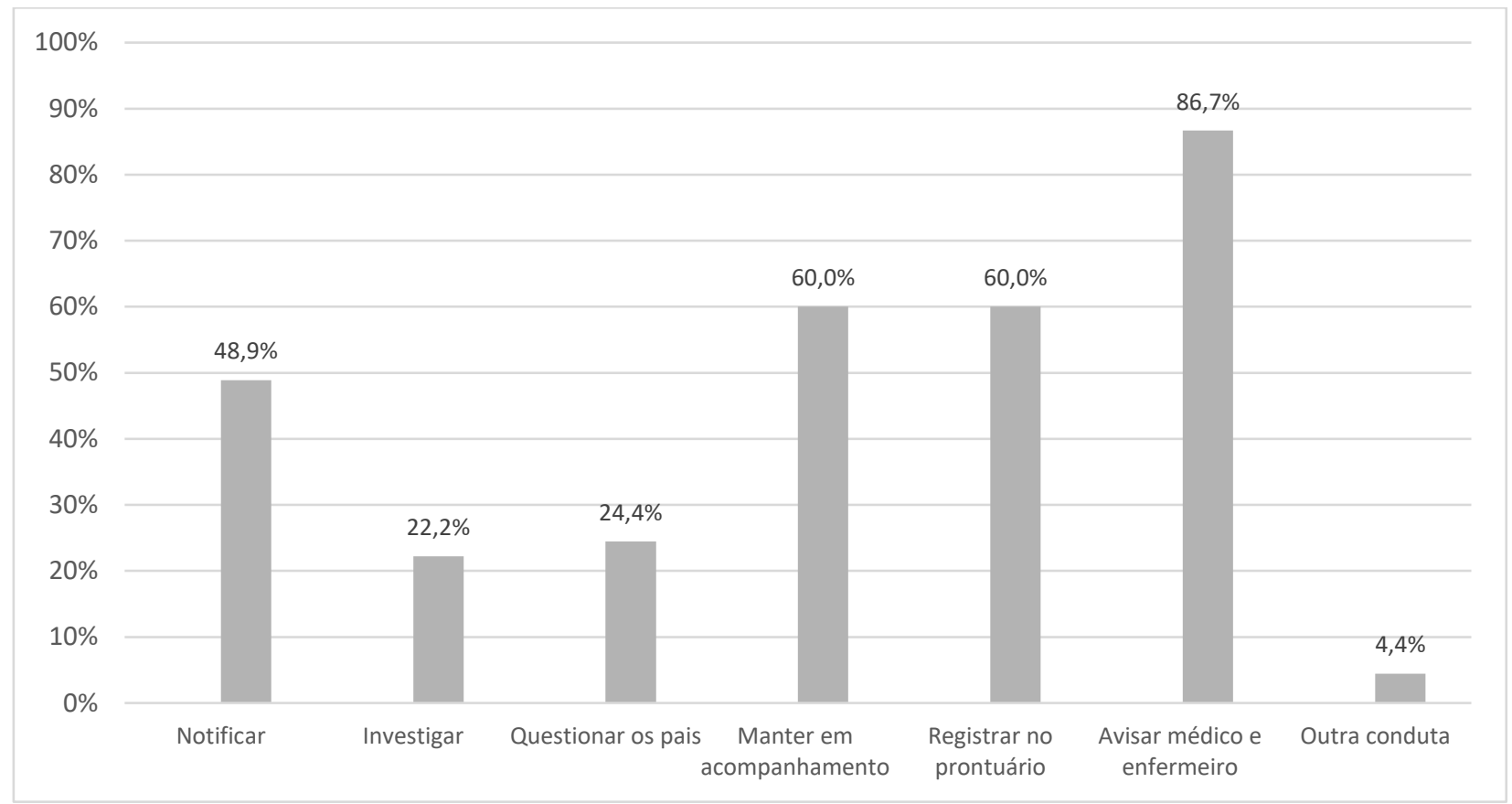

Gráfico 1. Distribuição da amostra segundo as condutas frente a casos suspeitos de maus-tratos infantis $(n=45)$

Tabela 3. Atitudes dos cirurgiões-dentistas da cidade de Pelotas frente a maus-tratos infantis $(n=45)$

\begin{tabular}{lcc}
\hline Itens do questionário & n & \% \\
\hline "Já suspeitou de algum caso?" & & \\
Não & 27 & 60,0 \\
Sim & 18 & 40,0 \\
"Foi o primeiro profissional a suspeitar?" & 15 & 33,3 \\
Não & 3 & 6,7 \\
Sim & 27 & 60,0 \\
Não se aplica (nunca suspeitou) & & \\
"Alguma atitude foi tomada?"* & 1 & 2,3 \\
Não & 11 & 25,0 \\
Sim & 5 & 11,4 \\
Não lembra & 27 & 61,3 \\
Não se aplica (nunca suspeitou) & 27 &
\end{tabular}


Tabela 4. Associação entre variáveis de caracterização do CD com seu conhecimento sobre maus-tratos infantis ( $\mathrm{n}=45$ )

\begin{tabular}{|c|c|c|c|c|c|c|c|c|c|c|c|c|c|c|c|}
\hline \multirow[t]{2}{*}{ Variável } & \multicolumn{5}{|c|}{$\begin{array}{l}\text { Responsabilidade do } \\
\text { cirurgião-dentista }\end{array}$} & \multicolumn{5}{|c|}{$\begin{array}{l}\text { Ação diante de suspeita de } \\
\text { maus-tratos infantis }\end{array}$} & \multicolumn{5}{|c|}{$\begin{array}{l}\text { Implicância legal da não notificação de } \\
\text { suspeita** }\end{array}$} \\
\hline & Incorreto & $\%$ & Correto & $\%$ & Valor-p & Incorreto & $\%$ & Correto & $\%$ & Valor-p & Incorreto & $\%$ & Correto & $\%$ & Valor- $\mathbf{p}$ \\
\hline Sexo & & & & & $0,020 !$ & & & & & $1,000^{!}$ & & & & & $0,370^{!}$ \\
\hline Feminino & 9 & 22,5 & 31 & 77,5 & & 20 & 50,0 & 20 & 50,0 & & 21 & 53,8 & 18 & 46,1 & \\
\hline Masculino & 4 & 80,0 & 1 & 20,0 & & 3 & 60,0 & 2 & 40,0 & & 4 & 80,0 & 1 & 20,0 & \\
\hline Idade (anos) & & & & & $0,094 *$ & & & & & $0,167 *$ & & & & & $0,049 *$ \\
\hline $28-42$ & 5 & 19,2 & 21 & 80,8 & & 11 & 42,3 & 15 & 57,7 & & 11 & 44,0 & 14 & 56,0 & \\
\hline $43-69$ & 8 & 42,1 & 11 & 57,9 & & 12 & 63,2 & 7 & 36,8 & & 14 & 73,7 & 5 & 26,3 & \\
\hline Tempo de formado & & & & & $0,019 !$ & & & & & $0.175^{*}$ & & & & & $0,013 *$ \\
\hline Até 16 anos & 3 & 12,5 & 21 & 87,5 & & 10 & 41,7 & 14 & 58,3 & & 9 & 39,1 & 14 & 60,9 & \\
\hline Maior que 16 anos & 10 & 47,6 & 11 & 52,4 & & 13 & 61,9 & 8 & 38,1 & & 16 & 76,2 & 5 & 23,8 & \\
\hline Modelo de Atenção da L & & & & & $0,081^{!}$ & & & & & $0,565^{*}$ & & & & & $0,248^{!}$ \\
\hline ESF & 2 & 13,3 & 13 & 86,7 & & 6 & 40,0 & 9 & 60,0 & & 10 & 71,4 & 4 & 28,6 & \\
\hline Misto & 9 & 47,4 & 10 & 52,6 & & 11 & 57,9 & 8 & 42,1 & & 11 & 57,9 & 8 & 42,1 & \\
\hline Tradicional & 2 & 18,2 & 9 & 81,8 & & 6 & 54,6 & 5 & 45,4 & & 4 & 36,4 & 7 & 63,6 & \\
\hline Dentistas pertencentes a & eSF & & & & $0,311^{!}$ & & & & & $0,299^{*}$ & & & & & $0,113^{!}$ \\
\hline Não/tradicional & 10 & 35,7 & 18 & 64,3 & & 16 & 57,1 & 12 & 42,9 & & 13 & 46,4 & 15 & 53,6 & \\
\hline Sim & 3 & 17,7 & 14 & 82,3 & & 7 & 41,2 & 10 & 58,8 & & 12 & 75,0 & 4 & 25,0 & \\
\hline Rotina de Trabalho & & & & & $0,287^{!}$ & & & & & $0,109^{!}$ & & & & & $0,335^{!}$ \\
\hline Integralmente público & 2 & 15,4 & 11 & 84,6 & & 4 & 30,8 & 9 & 69,2 & & 9 & 69,2 & 4 & 30,8 & \\
\hline Público/Privado & 11 & 34,4 & 21 & 65,6 & & 19 & 59,4 & 13 & 40,6 & & 16 & 51,6 & 15 & 48,4 & \\
\hline $\begin{array}{l}\text { Pós-graduação Stricto } \\
\text { Sensu }\end{array}$ & & & & & $0,467^{!}$ & & & & & $0,017^{!}$ & & & & & $0,164^{!}$ \\
\hline Não possui & 11 & 32,3 & 23 & 67,6 & & 21 & 61,8 & 13 & 38,2 & & 21 & 63,6 & 12 & 36,4 & \\
\hline Possui ou está cursando & 2 & 18,2 & 9 & 81,8 & & 2 & 18,2 & 9 & 81,8 & & 4 & 36,4 & 7 & 63,6 & \\
\hline
\end{tabular}


Conhecimentos e atitudes de cirurgiões-dentistas da rede pública de Pelotas-RS frente aos maus-tratos infantis

Tabela 5. Associação entre variáveis de caracterização do CD com a atitude frente a casos suspeitos de violência $(n=44)$

\begin{tabular}{|c|c|c|c|c|c|c|c|}
\hline \multirow{2}{*}{ Variável } & \multicolumn{7}{|c|}{ Tomada de atitude frente a caso suspeito de maus-tratos infantis** } \\
\hline & Incorreto & $\%$ & Correto & $\%$ & Nunca suspeitou & $\%$ & Valor-p \\
\hline Sexo & & & & & & & $1,000 !$ \\
\hline Feminino & 5 & 12,8 & 10 & 25,7 & 24 & 61,5 & \\
\hline Masculino & 1 & 20,0 & 1 & 20,0 & 3 & 60,0 & \\
\hline Idade (anos) & & & & & & & $0,912^{!}$ \\
\hline $28-42$ & 3 & 11,6 & 7 & 26,9 & 16 & 61,5 & \\
\hline $43-69$ & 3 & 16,7 & 4 & 22,2 & 11 & 61,1 & \\
\hline Tempo de formado & & & & & & & $1,000^{!}$ \\
\hline Até 16 anos & 3 & 12,5 & 6 & 25,0 & 15 & 62,5 & \\
\hline Maior que 16 anos & 3 & 15,0 & 5 & 25,0 & 12 & 60,0 & \\
\hline Modelo de Atenção da UBS & & & & & & & $0,644^{!}$ \\
\hline ESF & 2 & 14,3 & 5 & 35,7 & 7 & 50,00 & \\
\hline Misto & 2 & 10,5 & 3 & 15,8 & 14 & 73,7 & \\
\hline Tradicional & 2 & 18,2 & 3 & 27,3 & 6 & 54,5 & \\
\hline Dentistas pertencentes a ESF & & & & & & & $0,900^{!}$ \\
\hline Não/tradicional & 4 & 14,3 & 6 & 21,4 & 18 & 64,3 & \\
\hline Sim & 2 & 12,5 & 5 & 31,3 & 9 & 56,2 & \\
\hline Rotina de Trabalho & & & & & & & $0,505^{!}$ \\
\hline Integralmente público & 1 & 7,7 & 2 & 15,4 & 10 & 76,9 & \\
\hline Público/Privado & 5 & 16,1 & 9 & 29,03 & 17 & 54,8 & \\
\hline Pós-graduação Stricto Sensu & & & & & & & $0,470^{!}$ \\
\hline Não possui & 4 & 12,1 & 10 & 30,3 & 19 & 57,6 & \\
\hline Possui ou está cursando & 2 & 18,2 & 1 & 9,1 & 8 & 72,7 & \\
\hline
\end{tabular}

**Dados perdidos $\quad$ 'Valor p obtido pelo Teste Exato de Fisher 


\section{DISCUSSÃO}

A avaliação e análise dos conhecimentos e atitudes dos CD da rede municipal de Pelotas-RS frente aos MTI permite que seja possível traçar um perfil da atenção à saúde relativa a esta temática. Assim, são oferecidos subsídios para investigar as possíveis falhas no reconhecimento do papel que os profissionais representam frente aos casos suspeitos de MTI e também na conduta que tomam para seu combate. A partir dos resultados deste estudo, percebeu-se que a maior parte dos $\mathrm{CD}$ da rede pública de saúde do município atuante nas UBS, reconhece sua responsabilidade na identificação de casos de MTI, porém apresentam dificuldades quanto a condução dos casos, bem como desconhecem a implicância legal aplicada aos profissionais que não tomam atitudes frente a uma suspeita.

Verificou-se também que o tempo de formado teve associação com duas perguntas relacionadas ao conhecimento do $\mathrm{CD}$, sendo a resposta correta mais prevalente para aqueles com menor tempo de formado, tanto para o conhecimento da responsabilidade do $\mathrm{CD}$ na identificação dos MTI, quanto para o conhecimento da implicância legal. Estes achados podem revelar uma melhor abordagem desta temática nos últimos anos ${ }^{18}$; ou mesmo como resultado da presença desta temática nas mídias sociais, que são capazes de levar informações à toda a população ${ }^{19}$.

Adicionalmente, essa associação pode ter relação com o fato de este tema ter sido incluído no conteúdo da graduação da Faculdade de Odontologia da Universidade Federal de Pelotas a partir da reestruturação curricular que ocorreu em 2003, pois estima-se que a maior parte dos $\mathrm{CD}$ da rede municipal graduou-se nessa instituição. A mesma mudança de paradigma também pode ter ocorrido em outras instituições de ensino a partir de 2002, quando as Diretrizes
Curriculares Nacionais (DCN), do curso de graduação em Odontologia, foram atualizadas pela Resolução CNE/CES $n^{\circ}$ 3, de 19 de fevereiro de 2002, enfatizando a formação de um profissional crítico, humanista, reflexivo, de forma abrangente e que promova a integração dos cuidados de saúde ${ }^{20}$. Isso incentiva discussões de diversas temáticas, entre as quais os MTI, sendo um fator que pode ter contribuído para a mudança curricular nas instituições de ensino superior, de forma a incluir algum tipo de informação sobre esse tema.

Aproximadamente 1/3 dos profissionais relatou ter recebido informações sobre este assunto na graduação. A falta de informações nos cursos de graduação sobre os MTI é bastante citada na literatura ${ }^{15,21-24}$, mas mesmo em cursos que abordam a temática identifica-se a falta de preparo dos profissionais para lidar com estes $\operatorname{casos}^{25}$. Estudos demonstram que há uma parcela de profissionais que recebe essa informação durante o curso de graduação e na pósgraduação, mas destacam que apenas o ato de receber essas informações não parece ser suficiente para resultar em boa conduta frente aos casos suspeitos de MTI. Demonstra-se assim, a necessidade de incluir o tema nas ações de Educação Permanente em Saúde para profissionais da Odontologia, dando-lhes suporte na sua atuação profissional ${ }^{26-29}$. Entretanto, podese constatar que essa não é uma prática no serviço, uma vez que apenas um CD afirmou ter recebido capacitação sobre MTI.

Em virtude do tipo de formação requerida na graduação, se torna explicíta a necessidade e importância de trabalhar com a temática dos MTI, que ainda é um assunto abrangido de forma muito introdutória e principalmente no final na formação ${ }^{30}$. Pensando na importância de desenvolver a discussão do tema durante toda a graduação, Ivanoff e Hottel (2013) ${ }^{31}$ propuseram 
um currículo híbrido e multidisciplinar com dinâmicas de aprendizagem baseada no problema, dividido em 4 fases diferentes, abrangendo desde etapas como a introdução sobre as características da violência, associação de palestras e discussão de casos sobre o tema até simulação da notificação às autoridades e o exercício na prática ${ }^{31}$. Busca-se dessa forma que a formação em Odontologia seja mais abrangente.

Apesar de uma boa parcela da amostra se considerar de certa forma capaz de identificar MTI, corroborando com os achados de Azevedo et al. $(2012)^{32}$, outros estudos demonstram que existe subnotificação dos casos por profissionais de saúde, o que pode expor a existência de uma lacuna entre a identificação dos casos e sua notificação ${ }^{8,32-36}$. Mesmo considerando-se capaz de identificar um caso suspeito, muitos $C D$ não sabem como proceder. A reduzida taxa de notificações pode então estar associada não só à falta de conteúdo sobre essa temática nos currículos dos cursos de graduação e à falta de treinamento, mas também ao receio de realizar notificação e falta de conhecimento sobre o amparo legal que os $\mathrm{CD}$ possuem para esses $\operatorname{casos}^{8,26,37}$. A partir das respostas dos CD, parece haver uma transferência de responsabilidades para os outros profissionais que atuam na equipe da UBS.

Sobre a competência geral da liderança, as DCN recomendam que "no trabalho em equipe multiprofissional, os profissionais de saúde deverão estar aptos a assumirem posições de liderança, sempre tendo em vista o bem estar da comunidade. A liderança envolve compromisso, responsabilidade, empatia, habilidade para tomada de decisões, comunicação e gerenciamento de forma efetiva e eficaz, ${ }^{, 20}$. Todavia, esse contexto parece ser pouco abordado no currículo da graduação, o que pode levar a essas atitudes e omissões observadas no presente estudo.

Ademais, no estudo de Luna et al. $(2010)^{38}$, observou-se associação estatística entre ter cursado pós-graduação (em todas as suas modalidades, incluindo também a especialização e a residência) e o conhecimento sobre a necessidade de notificação, pois os profissionais estiveram mais envolvidos com os casos, o que pode corroborar com os achados do presente estudo, que avaliou apenas se o CD tinha cursado mestrado e/ou doutorado. Esse fato possivelmente pode se dar devido ao perfil dos CD que buscam a pós-graduação, os quais são profissionais motivados a sempre renovar seus conhecimentos, ou por terem mais contato com professores, colegas de profissão e aulas sobre a temática, o que pode tornar mais acessível a informação.

A Atenção Primária caracteriza-se como espaço importante para o reconhecimento dos casos suspeitos de MTI, por ser considerada como a porta de entrada dos indivíduos no serviço de saúde e também um espaço onde os profissionais atuam em equipes, fornecendo uma articulação necessária para combater os $\mathrm{MTI}^{39}$. Portanto, a transferência da responsabilidade sobre os casos pode também ser favorecida pelo trabalho em equipe da UBS.

Durante a formação em Odontologia deseja-se que o estudante tenha os conhecimentos necessários para exercer a competência geral de liderança na profissão, para assumir a posição de líder nas equipes multiprofissionais, para a tomada de atitude ${ }^{20}$. Dessa forma, pode-se observar que é importante que o enfrentamento dos MTI seja uma prática interprofissional e focada no cuidado, mas que o CD entenda que ele também tem amparo legal no processo de notificação e que está apto a realizar a condução dos casos em conjunto com a equipe.

A ficha de notificação de violência permite o detalhamento do registro de informações fiéis 
sobre a ocorrência e deve estar acessível nas rotinas das instituições. Por meio desta notificação compulsória é que se torna possível conhecer a real prevalência e a gravidade dos casos, definindo-se assim a forma adequada das ações de enfrentamento do problema. Entretanto, o presente estudo observou que nenhum profissional conhecia esta ficha, corroborando com achados de estudos prévios ${ }^{37,40}$. Esta falta de conhecimento pode estar relacionada à gestão e organização do serviço, pois como faz parte da atenção à saúde, a ficha de notificação, assim como os demais registros do processo de trabalho, deveria ser apresentada ao profissional. Pode, também, ocorrer devido ao entendimento de que o papel de registro não cabe ao $\mathrm{CD}$, mas a outros membros da equipe, como o assistente social e o enfermeiro. Pode, ainda, haver o desinteresse do CD em buscar saber mais sobre o tema e as condutas corretas a se tomar.

A estratégia da Educação Permanente em Saúde traz o SUS como uma aprendizagem rotineira e coletiva, sendo os profissionais os principais responsáveis pelo acolhimento, cuidado e intervenções, de forma a realizar um trabalho qualificado e resolutivo ${ }^{41}$. Nesse contexto, é importante que se tenha a Educação Permanente em Saúde sobre os MTI, para o conhecimento de todos os processos.

Os efeitos da feminização da profissão ainda não são totalmente claros, todavia tem sido relatado por estudos que a relação entre paciente e a profissional do sexo feminino possui maior empatia, carinho e melhor comunicação. Sendo assim, essas profissionais são consideradas mais humanas, atenciosas e cuidadosas ${ }^{42,43}$. Estes fatores podem explicar a associação observada no presente estudo, visto que as mulheres tiveram maior percepção das responsabilidades da profissão frente à violência.

Entre as limitações do estudo destaca-se que a coleta de dados foi realizada por meio de questionário autorrespondido, ou seja, dependente da plena compreensão para o preenchimento completo de todas as questões e da vontade dos participantes. Além disso as respostas podem ter sido dadas conforme aceitação social, ou seja, buscando a resposta melhor aceita e não a que de fato ocorre, apesar do sigilo garantido aos participantes. Outra questão importante é que estes dados refletem o conhecimento e atitudes dos CD da rede pública do município de Pelotas, não permitindo extrapolar estes achados para os profissionais de outros municípios e regiões, pois a realidade pode ser bem diferente.

Portanto, o fortalecimento desta temática nos currículos de graduação em Odontologia e o contínuo treinamento interprofissional, de forma a promover uma Educação Permanente da temática nos serviços em saúde, são medidas essenciais para melhorar a compreensão e a identificação de casos suspeitos de MTI, visando seguir as condutas adequadas e preconizadas por lei.

\section{CONCLUSÃO}

Ainda que os profissionais reconheçam que é responsabilidade do CD identificar casos de MTI, percebeu-se certa dificuldade referente às atitudes diante desta violência. Não houve associação entre a atitude frente a caso suspeito de MTI e o processo de trabalho. Contudo, constatou-se maior conhecimento entre os CD formados mais recentemente, sugerindo que a temática MTI esteja mais presente na sua formação e atuação.

\section{ABSTRACT \\ Knowledge and attitudes of dentists in the public health system of Pelotas-RS regarding child maltreatment}

The present study aimed to identify and analyze the knowledge and attitudes of dentists in the public health system in Pelotas-RS regarding child maltreatment $(\mathrm{CM})$. Data were collected 
through semi-structured questionnaire. Sociodemographic information, work-related data and knowledge and attitudes of dentists were collected. Descriptive statistical analysis and associations between independent variables and knowledge and attitudes of dentists were performed using Fisher's Exact Test and ChiSquare Test $(\mathrm{p}<0.05)$. Of the 45 dentists included in the sample, more than half reported that they had never received information on the subject, $71.1 \%$ understood the dentist's responsibility in the identification of suspicious cases, $50 \%$ were unable to inform about the legal implication of the lack of notification and $86.7 \%$ believed that the doctor or nurse should be notified so that they can take action. It was observed that $60 \%$ of professionals never suspected a case of $\mathrm{CM}$ and $25 \%$ of those who suspected took action. There was statistical association between the dentist's responsibility and gender and the time since graduation, between correct attitude towards a suspicious case and postgraduate degree and also between legal implication with age and time since graduation. Knowledge and attitudes of dentists about CM were better among younger professionals, females, with less time since graduation, having or attending some postgraduate course. Dentists know their importance towards this violence, but they show difficulties regarding attitudes that they should take.

Descriptors: Child Abuse. Violence. Forensic Dentistry. Education, Dental.

\section{REFERÊNCIAS}

1. Brasil. Lei n. 8069, de 13 de julho de 1990. Brasília: Presidência da República; 1990; [Acesso em: 20 de março de 2020]. Disponível em: http://www.planalto.gov. br/ccivil_03/leis/18069.htm.

2. da Silva-Junior IF, Hartwig AD, Demarco GT, Stuermer VM, Scobernatti G, Goettems ML, Azevedo MS. Health-related quality of life of maltreated children and adolescents who attended a service center in Brazil. Qual Life Res. 2018;27(8):2157-64.
3. Cavalcanti AL. Prevalence and characteristics of injuries to the head and orofacial region in physically abused children and adolescents--a retrospective study in a city of the Northeast of Brazil. Dent Traumatol. 2010;26(2):149-53.

4. da Silva-Junior IF, Hartwig AD, Stuermer VM, Demarco GT, Goettems ML, Azevedo MS. Oral health-related quality of life in Brazilian child abuse victims: A comparative study. Child Abuse Negl. 2018;76:452-8.

5. Leeb R, Paulozzi L, Melanson C, Simon T, Arias I. Child Maltreatment Surveillance: Uniform Definitions for Public Healthand Recommended Data Elements Version 1.0 ed. Atlanta: National Center for Injury Prevention and Control; 2008.

6. da Silva Franzin LC, Olandovski M, Vettorazzi ML, Werneck RI, Moyses SJ, Kusma SZ, Moyses ST. Child and adolescent abuse and neglect in the city of Curitiba, Brazil. Child Abuse Negl. 2014;38(10):170614.

7. Massoni AC, Ferreira AM, Aragao AK, de Menezes VA, Colares V. Aspectos orofaciais dos maus-tratos infantis e da negligência odontológica. Cien Saude Colet. 2010;15(2):403-10.

8. Clarke L, Chana P, Nazzal H, Barry S. Experience of and barriers to reporting child safeguarding concerns among general dental practitioners across Greater Manchester. $\mathrm{Br}$ Dent J. 2019;227(5):387-91.

9. Sousa GFP, Carvalho MMP, GrainvilleGarcia AF, Gomes MNC, Ferreira JMS. Conhecimento de acadêmicos em odontologia sobre maus-tratos infantis. Odonto. 2012;20(40):101-8.

10. Yehuda Y, Attar-Schwartz S, Ziv A, Jedwab M, Benbenishty R. Child abuse and neglect: reporting by health professionals and their need for training. Isr Med Assoc J. 
2010;12(10):598-602.

11. Brasil. Notificação de maus-tratos contra crianças e adolescentes pelos profissionais de saúde. Brasília: Ministério da Saúde; 2002; [Acesso em: 19 de março de 2020]. Disponível em: http://bvsms.saude.gov.br/ bvs/publicacoes/notificacao_maustratos_cri ancas_adolescentes.pdf.

12. Garbin CA, Dias Ide A, Rovida TA, Garbin AJ. Desafios do profissional de saúde na notificação da violência: obrigatoriedade, efetivação e encaminhamento. Cien Saude Colet. 2015;20(6):1879-90.

13. Garcia L, Maio I, Santos T, Folha C, Watanabe $\mathrm{H}$. Intersetorialidade na saúde no Brasil no início do século XXI: um retrato das experiências. Saúde Debate. 2014;38(103):966-80.

14. Maia JN, Ferrari RAP, Gabani FL, Tacla MTGM, Reis TB, Fernandes MLC. Violência contra criança: cotidiano de profissionais na atenção primária à saúde. Rev Rene. 2016;17(5):593-601.

15. Matos FZ, Borges AH, Neto IM, Rezende CD, Silva KL, Pedro FLM, Porto AN. Avaliação do conhecimento dos alunos de graduação em odontologia x cirurgião dentista no diagnóstico de maus-tratos a crianças. Rev Odontol Bras Central. 2013;22(63).

16. Cavalcanti AL, Martins VM. Percepções e Conhecimentos de Médicos Pediatras e Cirurgiões-Dentistas Sobre Maus-Tratos Infantis. RBCS. 2009;13(3):41-8.

17. Santos J, Yakuma M. A Estratégia Saúde da Família frente à violência contra crianças: revisão integrativa. Revista da Sociedade Brasileira de Enfermeiros Pediatras. 2015;15(1):38-43.

18. Nikolic S, Zivkovic V. Child maltreatment and neglect, or poverty and ignorance: An old case from the museum. Med Sci Law. 2016;56(2):150-3.
19. Schwab-Reese LM, Hovdestad W, Tonmyr L, Fluke J. The potential use of social media and other internet-related data and communications for child maltreatment surveillance and epidemiological research: Scoping review and recommendations. Child Abuse Negl. 2018;85:187-201.

20. Brasil. Resolução CNE/CES 3, de 19 de fevereiro de 2002: Institui Diretrizes Curriculares Nacionais do Curso de Graduação em Odontologia. Brasília: Conselho Nacional de Educação; 2002; [Acesso em: 22 de março de 2021]. Disponível em: http://portal.mec.gov.br/cne/ arquivos/pdf/CES032002.pdf.

21. Silva KBG, Cavalcanti AFC, Cavalcanti AL. Maus-tratos infantis: conhecimentos e condutas dos cirurgiões-dentistas da Estratégia Saúde da Família de Guarabira-PB, Brasil. REFACS. 2017;5(1):108-17.

22. Fracon ET, Silva RHA, Bregagnolo JC. Avaliação da conduta do cirurgião-dentista ante a violência doméstica contra crianças e adolescentes no município de Cravinhos (SP). RSBO. 2011;8(2):153-9.

23. Massoni ACLT, Almeida MANF, Martins CG, Firmino RT, Grainville-Garcia AF. Maus-tratos na infância e adolescência: conhecimento e atitude de profissionais de saúde. Arq Odontol. 2014;50(2):71-7.

24. Moura AR, Amorim A, Proença L, Milagre V. Dentists and undergraduate dental students require more information relating to child abuse. MedicalExpress. 2015;2(2).

25. Koifman L, Menezes RM, Bohrer KR. Abordagem do Tema "Violência contra a Criança" no Curso de Medicina da Universidade Federal Fluminense. Rev Bras Educ Med. 2012;36(2):172-9.

26. Condori PLP, Nascimento SCL, Mitie ABD, Pizzato E, Mazza VA, Buffon MCM. Maustratos na infância e adolescência: percepção e 
conduta de profissionais de nível superior que atuam na Estratégia Saúde da Família. RSBO. 2018;15(1):34-40.

27. Al Hajeri H, Al Halabi M, Kowash M, Khamis AH, Welbury R, Hussein I. Assessment of the knowledge of United Arab Emirates dentists of Child Maltreatment, protection and safeguarding. Eur J Paediatr Dent. 2018;19(2):105-18.

28. Brattabo IV, Bjorknes R, Breivik K, Astrom AN. Explaining the intention of dental health personnel to report suspected child maltreatment using a reasoned action approach. BMC Health Serv Res. 2019;19(1):507.

29. Jakobsen U, Fjallheim AS, Gislason $H$, Gudmundsen E, Poulsen S, Haubek D. Dental professionals' experience with and handling of suspicion of child maltreatment in a smallscale society, the Faroe Islands. Clin Exp Dent Res. 2019;5(2):145-50. Epub 2019/05/03.

30. Biss S, Duda J, Tomazinho P, Pizzatto E, Losso E. Maus tratos infantis: avaliação do currículo dos cursos em odontologia. Revista da ABENO. 2015;15(1):55-62.

31. Ivanoff $\mathrm{C}$, Hottel T. Comprehensive Training in Suspected Child Abuse and Neglect for Dental Students: A Hybrid Curriculum. Journal of Dental Education. 2013;77(6):695705 .

32. Azevedo MS, Goettems ML, Brito A, Possebon AP, Domingues J, Demarco FF, Torriani DD. Child maltreatment: a survey of dentists in southern Brazil. Braz Oral Res. 2012;26(1):5-11.

33. Silva-Oliveira F, Andrade CI, Guimaraes MO, Ferreira RC, Ferreira EF, Zarzar PM. Recognition of child physical abuse by a group of Brazilian primary care health professionals. Int J Paediatr Dent. 2019;29(5):624-34. Epub 2019/05/10.

34. Silva-Oliveira F, Ferreira e Ferreira E, Mattos
Fde F, Ribeiro MT, Cota LO, Vale MP, Zarzar

PM. Adaptação transcultural e reprodutibilidade de questionario para avaliação de conhecimento e atitude de profissionais de saude frente a casos de abuso fisico infantil. Cien Saude Colet. 2014;19(3):917-29.

35. Silva-Oliveira F, Andrade CI, Guimaraes MO, Ferreira RC, Ferreira e Ferreira E, Zarzar PM. Frequência de identificação e notificação de abuso físico infantil por profissionais da Estratégia Saúde da Família e relação com fatores socioeconômicos. Arq Odontol. 2017;53(9).

36. Ronneberg A, Nordgarden $H$, Skaare AB, Willumsen T. Barriers and factors influencing communication between dental professionals and Child Welfare Services in their everyday work. Int J Paediatr Dent. 2019;29(6):684-91.

37. Garbin CA, Rovida TA, Costa AA, Garbin AJ. Percepção e atitude do cirurgião-dentista servidor público frente à violência intrafamiliar em 24 municípios do interior do estado São Paulo, 2013-2014. Epidemiol Serv Saúde. 2016;25(1):179-86.

38. Luna GL, Ferreira RC, Vieira LJ. Notificação de maus-tratos em crianças e adolescentes por profissionais da Equipe Saúde da Familia. Cien Saude Colet. 2010;15(2):481-91.

39. Algeri S, Souza LM. Violência Contra Crianças E Adolescentes: Um Desafio No Cotidiano Da Equipe De Enfermagem. Rev Latino-Am Enfermagem. 2006;14(4).

40. Moreira GAR, Rolim ACA, Saintrain MVL, Vieira LJES. Atuação do cirurgião-dentista na identificação de maus-tratos contra crianças e adolescentes na atenção primária. Saúde Debate. 2015;39:257-67.

41. Brasil. Educação Permanente em Saúde: Reconhecer a produção local de cotidianos de saúde e ativar práticas colaborativas de aprendizagem e de entrelaçamento de 
saberes. Brasília: Ministério da Saúde; 2014;

[Acesso em: 23 de março de 2021].

Disponível em: http://bvsms.saude.gov.br/ bvs/folder/educacao_permanente_saude.pdf.

42. McKay JC, Quinonez CR. The feminization of dentistry: implications for the profession. J Can Dent Assoc. 2012;78:c1.

43. Kfouri MG, Moyses SJ, Moyses ST. Women's motivation to become dentists in Brazil. J Dent Educ. 2013;77(6):810-6.

\section{Correspondência para:}

Marina Sousa Azevedo

e-mail: marinasazevedo@gmail.com

Universidade Federal de Pelotas

Faculdade de Odontologia

Departamento de Odontologia Social e

Preventiva

Rua Gonçalves Chaves, 457, Centro - $7^{\circ}$ andar

96015-560 Pelotas/RS 